A-11

\title{
TRANSPIRACIÓN NOCTURNA EN VID
}

\author{
Montoro, A. ${ }^{(1)}$; López-Urrea, R. ${ }^{(2)}$; Mañas, F. ${ }^{(3)}$; Martínez, L. ${ }^{(4)}$; Sánchez, J.M. ${ }^{(5)}$
}

1 Investigadora, ITAP, Polígono Campollano, Avda. 2a -42 B, 02007 Albacete. meli.itap@dipualba.es

2 Director, ITAP-FUNDESCAM, Polígono Campollano, Avda. 2a -42 B, 02007 Albacete. rlu.itap@dipualba.es

${ }^{3}$ Técnico, ITAP, Polígono Campollano, Avda. 2a-42 B, 02007 Albacete.fmj.itap@dipualba.es

4 Técnica, FUNDESCAM, Polígono Campollano, Avda. 2a -42 B, 02007 Albacete. Imm.itap@dipualba.es

5 Profesor, UCLM, Dpto. Física Aplicada, E.U. Politécnica de Cuenca, 16071 Cuenca, juanmanuel.sanchez@uclm.es

\section{Resumen}

Se presentan las medidas de transpiración media cada quince minutos, de la vid (Vitis vinifera cv. Tempranillo), en tres periodos fenológicos del cultivo: cerrado de racimo, envero y maduración. El estudio se realizó en dos plantas de 16 años de edad, a un marco de plantación de $3 \mathrm{~m} \times 1,5 \mathrm{~m}$, regadas mediante goteo y plantadas en un lisímetro de pesada continua monolítico de $9 \mathrm{~m}^{2}$ de superficie. Con la finalidad de poder medir la transpiración, la superficie del lisímetro fue cubierta con una lona impermeable, para evitar la pérdida de agua por evaporación, dejándose únicamente al aire libre el cultivo; de esta manera, las medidas registradas de pérdida de peso se debieron únicamente al efecto de la transpiración. Los resultados indicaron transpiración antes y después de la salida del sol, oscilando los valores de un $7 \%$ a un $16 \%$, con respecto a la transpiración total del día.

\section{1- Introducción y objetivos}

La transpiración (T) determina el estado hídrico de la planta. Este proceso comprende la evaporación del agua desde las células superficiales en el interior de los espacios intercelulares y su difusión fuera del tejido vegetal. Esta difusión la realizan principalmente a través de los estomas, los cuales se abren para captar $\mathrm{CO}_{2}$ y en ese proceso se produce la pérdida de vapor de agua, debido en parte a su menor peso molecular ( $18 \mathrm{~g} / \mathrm{mol}$ de la molécula de agua, frente a $44 \mathrm{~g} / \mathrm{mol}$ del anhídrido carbónico) y en gran medida al fuerte gradiente de diferencia de presión de vapor (DPV) entre el aire y la hoja. Por tanto, los estomas ejercen el mayor control a corto plazo en las relaciones hídricas de una planta (Squeo y Leon, 2007).

La conductancia estomática y la $\mathrm{T}$ se ven afectadas por los factores externos medioambientales que determinan la demanda evaporativa de la atmósfera, entre los que destacan la temperatura, radiación solar, velocidad de viento y humedad relativa del aire. Junto a estos factores, el contenido de humedad del suelo y su mayor o menor facilidad para ponerla a disposición de la planta son determinantes en el proceso de T. En tercer lugar, un complejo y gran número de variables biológicas intervendrán en el proceso de la $\mathrm{T}$ : respuestas hormonales a nivel radicular, señales de conductividad hidráulica del xilema (Sperry et al., 1998) y control estomático. Por último, todo lo referente a la arquitectura de la planta (altura del cultivo, cantidad y estructura foliar), determinará el proceso transpirativo.

Los mecanismos que subyacen en la apertura estomática y por tanto en la $\mathrm{T}$, no están totalmente aclarados, pues aunque usualmente la apertura de los estomas ocurre en presencia de luz, Sharkey y Raschke (1981) demostraron que a niveles bajos de radiación, 
la concentración de $\mathrm{CO}_{2}$ sería el principal factor regulador de la apertura estomática. Actualmente hay una controversia acerca de la apertura estomática nocturna y por tanto de la transpiración durante la noche en plantas C3 y C4.

El objetivo de este trabajo fue medir la transpiración diurna y nocturna en el cultivo de la vid, utilizando un lisímetro de pesada continua.

\section{2- Materiales y métodos}

El estudio se realizó en 2015, en la finca experimental "Las Tiesas" (Albacete, España), cuyas coordenadas geográficas son: $2^{\circ} 5^{\prime}$ longitud Oeste, $39^{\circ} 3^{\prime}$ latitud Norte, y $695 \mathrm{~m}$ sobre el nivel del mar. Las medidas se llevaron a cabo en vid (Vitis vinifera L., cv. Tempranillo), en dos cepas de 16 años de antigüedad plantadas en un lisímetro monolítico de pesada continua conducidas en espaldera, poda doble cordón y marco de plantación $3 \mathrm{x}$ $1,5 \mathrm{~m}$. El riego se aplicó por goteo, mediante una línea portagoteros con goteros autocompensantes de $4 \mathrm{l} / \mathrm{h}$ y 2 goteros por planta (López-Urrea et al., 2012) y de día. Las dimensiones del recipiente del lisímetro son de $3 \times 3 \mathrm{~m}$ de lado y $1,7 \mathrm{~m}$ de profundidad, conteniendo el cultivo en las mismas condiciones de desarrollo que el resto de la parcela de protección para que los datos sean representativos. El suelo del lisímetro se cubrió con una lona impermeable, con la finalidad de evitar el proceso evaporativo y poder medir directamente la $\mathrm{T}$ del cultivo cada 15 minutos. La T se calculó como la diferencia de las pérdidas de masa consecutivas registradas del lisímetro y repartidas entre toda la superficie del mismo $\left(9 \mathrm{~m}^{2}\right)$.

Con la finalidad de parametrizar el cultivo, se realizaron medidas a nivel de planta, éstas fueron la conductancia estomática $\left(g_{s}\right)$ y el potencial hídrico de hoja $\left(\Psi_{1}\right)$. Estas medidas se hicieron sobre hojas adultas elegidas del tercio medio de los pámpanos y a las 12 horas solares, la primera de ellas en las plantas del lisímetro y la segunda en vides de la parcela de protección, con la finalidad de no alterar las plantas del lisímetro, al ser el método de medida destructivo. La $\mathrm{g}_{\mathrm{s}}$ se midió con un porómetro modelo SC-1 (Decagon Devices Inc., Pullman, WA, USA) y el $\Psi_{1}$, con una cámara Scholander (PMS Instrument Company, Albany, Oregon, USA).

El desarrollo vegetativo del cultivo nos lo determinó el porcentaje de fracción de cubierta vegetal verde $\left(f_{c}\right)$. Para ello se instaló una cámara de vídeo en una estructura metálica de 4 metros de alta, anclada en los laterales exteriores del lisímetro. Se tomaron fotogramas diarios y posteriormente se clasificaron utilizando la técnica del algoritmo de máxima probabilidad con ayuda del programa informático ENVI®, obteniendo el porcentaje de cubierta vegetal verde y del suelo (Montoro, 2008).

El contenido de humedad del suelo fue medido de manera continua a 10, 40, 70 y $100 \mathrm{~cm}$ mediante sondas FDR (Frecuence Domain Reflectometry) (EnviroSCANTM, Sentek Pty Ltd., South Australia).

Las medidas atmosféricas se midieron en una estación meteorológica con medias de quince minutos, sincronizados estos con el reloj del lisímetro. Las medidas fueron de radiación solar (sensor piranómetro modelo CM14, Kipp \& Zonen Delft, Holland), velocidad del viento (anemómetro modelo A100R, Vector Instruments Ltd., UK), temperatura y humedad del aire (sensor PRT 100 Ohm e higrómetro C-80, respectivamente, ambas del modelo MP100, Campbell Scientific Instrument, Logan, UT, USA). La evapotranspiración de referencia $\left(\mathrm{ET}_{\mathrm{o}}\right.$ ) se calculó utilizando la ecuación de Penman-Monteith FAO56 (P-M FAO56) (Allen et al., 1998).

\section{3- Resultados y discusión}

La tabla 1 muestra los valores de evapotranspiración de referencia $\left(E T_{0}\right)$, riego aplicado y transpiración diurna y nocturna en los días que el lisímetro estuvo tapado con una lona impermeable, durante los estados fenológicos de cierre del racimo, envero y maduración, cuya fracción de cubierta vegetal verde era de 0.32 . En todos los días 
analizados se registró la T nocturna, oscilando ésta desde un $7 \%$ a un $16 \%$ del total diario, siendo en la mayoría de los días más elevada si ha venido seguida de horas de luz. Todo ello con independencia de la $\mathrm{ET}_{\text {o }}$ diaria registrada. Estos resultados están en la línea con los encontrados por otros autores recientemente (Caird et al., 2007 a, b; Novick et al., 2009; Escalona et al., 2013), viéndose comprometida la eficiencia del uso del agua en determinadas condiciones, en las que se favorecería una apertura estomática sin fotosíntesis asociada.

Tabla 1. Estado fenológico, fracción de cubierta vegetal verde, evapotranspiración de referencia $\left(E_{0}\right)$, riego aplicado y transpiración diurna y nocturna en los días que el lisímetro estuvo tapado con una lona impermeable.

\begin{tabular}{|c|c|c|c|c|c|c|c|c|c|}
\hline \multirow[t]{2}{*}{ Fecha } & \multirow{2}{*}{$\begin{array}{c}\text { Estado } \\
\text { fenológico }\end{array}$} & \multirow[t]{2}{*}{ fc } & \multirow{2}{*}{$\begin{array}{l}\text { ETo } \\
\text { (mm) }\end{array}$} & \multirow{2}{*}{$\begin{array}{l}\text { Riego } \\
(\mathrm{mm})\end{array}$} & \multirow{2}{*}{$\begin{array}{l}\text { T total } \\
(\mathrm{mm})\end{array}$} & \multicolumn{3}{|c|}{ T total al día (\%) } & \multirow{2}{*}{$\begin{array}{c}\text { T total } \\
\text { noche } \\
(\%)\end{array}$} \\
\hline & & & & & & noche & día & noche & \\
\hline 16-jul. & \multirow{2}{*}{$\begin{array}{c}\text { Cierre del } \\
\text { racimo }\end{array}$} & \multirow{2}{*}{0.32} & 8.72 & 4.68 & 2.34 & 2.6 & 92.7 & 4.7 & 7.3 \\
\hline 17-jul. & & & 8.78 & & 2.74 & 6.2 & 87.6 & 6.2 & 12.4 \\
\hline 30-jul. & \multirow{2}{*}{ Envero } & \multirow{2}{*}{0.32} & 7.35 & 5.16 & 1.83 & 1.1 & 84.1 & 14.8 & 15.9 \\
\hline 31-jul. & & & 9.14 & & 2.07 & 1.9 & 91.8 & 6.3 & 8.2 \\
\hline 13-ago. & \multirow{6}{*}{ Maduración } & \multirow{6}{*}{0.32} & 8.92 & 5.34 & 2.25 & 4.5 & 89.2 & 6.3 & 10.8 \\
\hline 14-ago. & & & 6.17 & & 2.11 & 6.2 & 87.2 & 6.6 & 12.8 \\
\hline 15-ago. & & & 5.79 & 5.74 & 1.94 & 2.1 & 88.1 & 9.8 & 11.9 \\
\hline 16-ago. & & & 4.81 & & 2.11 & 0.9 & 89.1 & 10.0 & 10.9 \\
\hline 17-ago. & & & 6.26 & 5.15 & 1.69 & 3.6 & 84.6 & 11.8 & 15.4 \\
\hline 18-ago. & & & 6.55 & & 2.97 & 1.7 & 89.2 & 9.1 & 10.8 \\
\hline
\end{tabular}

A modo de ejemplo se representa en la Figura 1 la evolución de los registros tomados cada 15 minutos del peso del lisímetro, así como la evolución de la transpiración calculada con dichos pesos, en uno de los días que estuvo tapado. Se observa el descenso continuo del peso, excepto de 8:15 a 8:45 (hora solar) en el que faltan 3 registros de datos por realizarse medidas en las plantas del lisímetro en ese intervalo. Las oscilaciones en la evolución de la transpiración cada quince minutos, corresponden a la mayor o menor pendiente que se produce entre dos medidas consecutivas de pesos.

Con el objeto de visualizar la transpiración nocturna, se muestra en la Figura 2 la transpiración junto con la radiación solar global cada cuarto de hora, destacando la existencia de transpiración de 0:00h a 4:45h y de 19:45h a 24:00h, horas en las que la radiación solar global no presenta valores positivos, por tanto, las medidas indican transpiración antes y después de la salida del sol en una noche de luna nueva, como se pone de manifiesto en la Figura 3, que representa la pérdida de peso nocturna en uno de los días, así como la transpiración calculada con la pérdida de peso. 


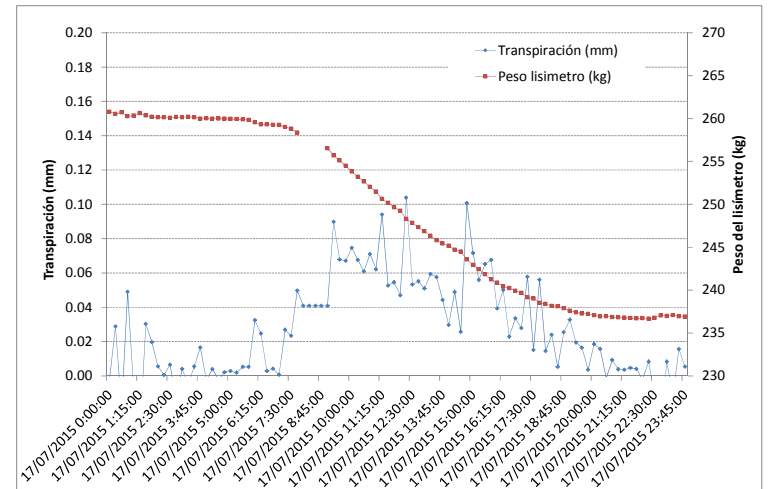

Figura 1. Evolución del peso del lisímetro y de la transpiración

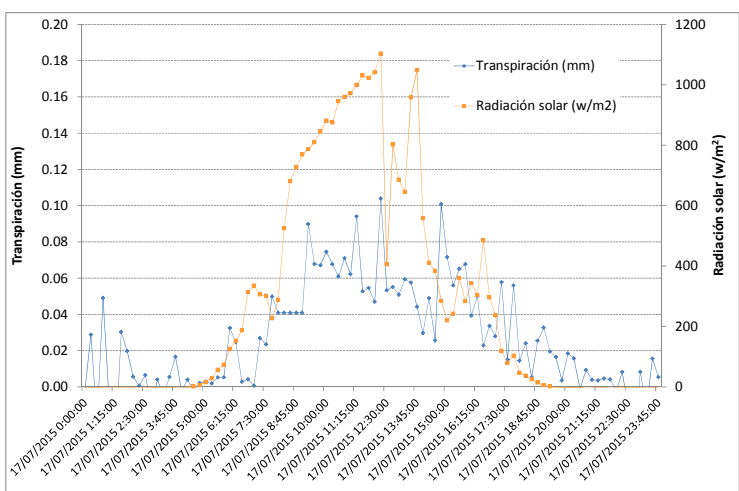

Figura 2. Evolución de la radiación solar frente a la transpiración medida en el lisímetro.

La relación encontrada en este trabajo entre transpiración y radiación solar presenta un $r^{2}$ de 0.40 (Figura 4), lo que parece indicar que aunque la radiación solar es uno de los parámetros externos que están más relacionados con la apertura estomática y por tanto con la transpiración, existen otros factores (tanto internos como externos) que determinan el proceso y serían los que están involucrados directamente en el proceso de transpiración nocturna.

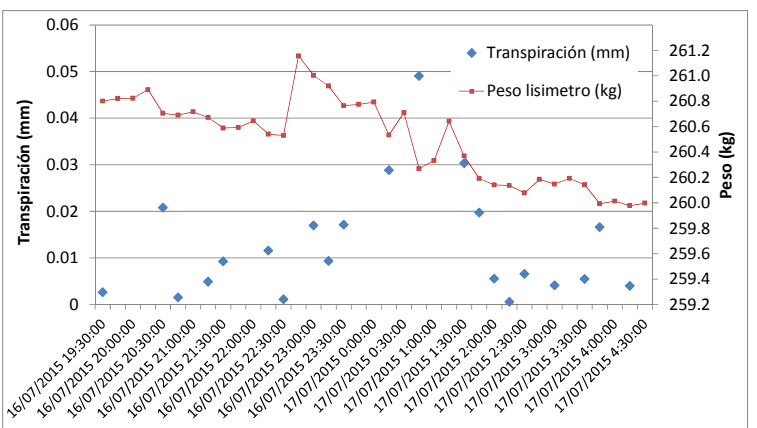

Figura 3. Evolución del peso del lisímetro y de la transpiración en la noche del 16 al 17 de julio.

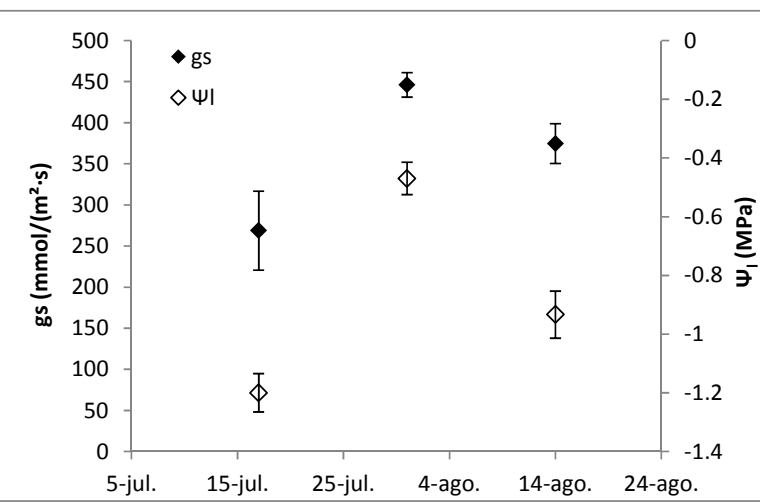

Figura 5. Potencial de hoja y conductancia estomática en cierre de racimo, envero y maduración.

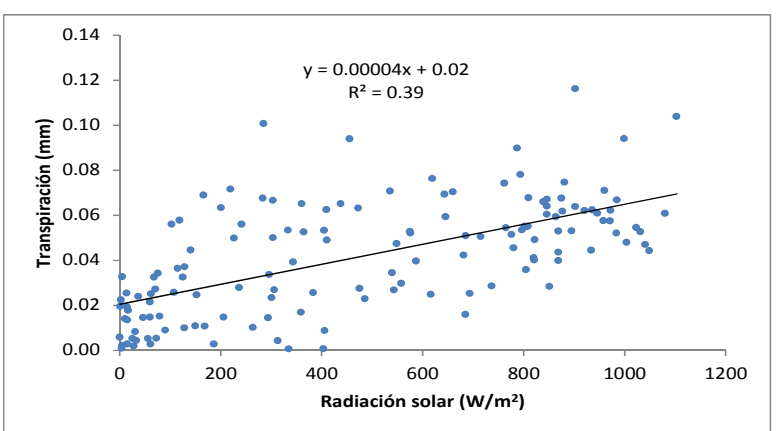

Figura 4. Relación entre radiación solar y transpiración.

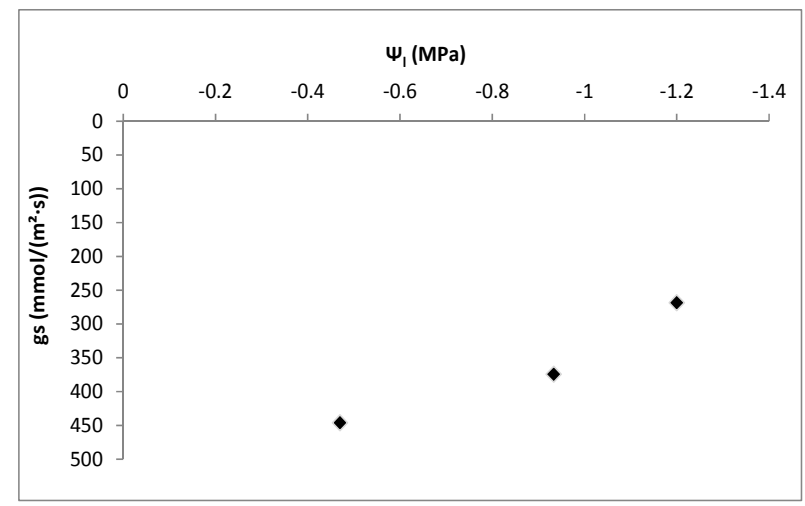

Figura 6. Relación entre potencial de hoja y conductancia estomática.

Por último, la Figura 5 representa el $\psi_{1} \mathrm{y} g_{s}$ en cierre del racimo, envero y maduración. Los valores de $\Psi_{1}$ indican que las plantas no presentaban estrés hídrico en envero, un estrés medio en maduración y el mayor estrés en el estado fenológico de cierre del racimo. Los valores de potencial para la valoración del estrés hídrico de las plantas se ha realizado teniendo en cuenta los resultados obtenidos en previos trabajos en el mismo área 
de estudio (Montoro et al., 2012) así como los obtenidos en otros estudios en diferentes áreas (Cifre et al., 2005; Sibille et al., 2007; Fuentes et al., 2014). La misma tendencia del potencial siguió la $\mathrm{g}_{\mathrm{s}}$. La Figura 6 muestra la relación entre ambos parámetros, siendo mayor $g_{s}$ cuanto mayor es $\Psi_{l}$, como era de esperar.

\section{4- Conclusiones y recomendaciones}

Los resultados indican la existencia de transpiración nocturna en plantas de Vitis vinifera $L$. en las condiciones ambientales y de régimen hídrico en el que se ha llevado el experimento.

\section{5.- Agradecimientos}

Agradecemos el apoyo económico obtenido del proyecto RTA2011-00100-C05-04 ya acabado y el actualmente vigente, el RTA 2014-00049-C05-03, así como el AGL201454201-C4-4-R, todos ellos financiados por el Ministerio de Economía y Competitividad, los dos primeros a través del Instituto Nacional de Investigación y Tecnología Agraria y Alimentaria y el último gracias al "Programa Estatal de Investigación, Desarrollo e Innovación Orientada a los Retos de la Sociedad"

\section{6- Bibliografía}

Allen, R.G., Pereira, L.S., Raes, D., Smith, M. (1998). Crop evapotranspiration. Guidelines for computing crop water requirements. FAO Irrigation and Drainage, paper $n^{\circ} .56$, FAO, Rome.

Caird, M.A., Richards, J.H., Donovan, L.A. (2007a). Nighttime Stomatal Conductance and Transpiration in C3 and C4 Plants. Plant Physiology 143:4-10.

Caird, M.A., Richards, J.H., Hsiao, T.C. (2007b). Significant transpiration and water loss occurs throughout the night in field-grown tomato. Functional Plant Biology 34, 172177.

Cifre, J., Bota, J., Escalona, J.M., Medrano, H., Flexas, J. (2005). Physiological tools for irrigations scheduling in grapevines (Vitis vinifera L.). An open gate improve water-use efficiency?. Agriculture, Ecosystems \& Environment 106, 159-170.

Escalona, J.M., Fuentes, S., Tomás, M., Martorell, S., Flexas, J., Medrano, H. (2013). Responses of leaf night transpiration to drought stress in Vitis vinifera L. Agricultural Water Management 118, 50-58.

Fuentes, S., de Bei, R., Collins, M.J., Escalona, J.M., Medrano, H., Tyerman, S. (2014). Night-time responses to water supply in grapevines (Vitis vinifera L.) under deficit irrigation and partial root-zone drying. Agricultural Water Management 138, 1-9.

López-Urrea, R., Montoro, A., Mañas, F., López-Fuster, P., Fereres, E. (2012). Evapotranspiration and crop coefficients from lysimeter measurements of mature "Tempranillo" wine grapes. Agricultural Water Management 112, 13-20.

Montoro, A. (2008). Avances en el Manejo del Agua en la Agricultura de Riego. Tesis Doctoral presentada en la ETSIA de la Universidad de Castilla-La Mancha.

Montoro, A., Fereres, E., López-Urrea, R., Mañas, F., López-Fuster, P. (2012). Sensitivity of trunk diameter fluctuations in Vitis vinifera L. Tempranillo and Cabernet Sauvignon Cultivars. American Journal of Enology and Viticulture 63 (1), 85-93.

Novick, K.A., Oren, R., Stoy, P.C., Siqueira, M.B.S., Katul, G.G. (2009). Nocturnal evapotranspiration in Eddy-covariance records from three co-located ecosystems in the Southeastern U.S.: Implications for anual fluxes. Agricultural and Forest Meteorlogy $149,1491-1504$. 
Sharkey, T.D. \& Raschke, K. (1981). Separation and measurement of direct and indirect effects of light on stomata. Plant Physiology 68: 33-40.

Sibille, I., Ojeda, H., Prieto, J., Maldonado, S., Lacapere, J.N., Carbonneau, A. (2007). Relation between the values of three pressure chamber modalities (midday leaf, midday stem and predawn water potential) of 4 grapevine cultivars in drought situation of the southern of France. Applications for the irrigation control. In: Proceedings of XVth Conference GESCO, Porec, Croatia, pp. 685-695.

Sperry J.S., Adler F.R., Campbell, G.S., Comstock, J.P. (1998). Limitation of plant water use by rhizosphere and xylem conductance: results from a model. Plant, Cell and Environment, 21: 347-359.

Squeo, F.A. y León, M. F. (2007). Capítulo III. Transpiración. En Fisiologia Vegetal. Squeo \& Cardemil, eds. Ediciones Universidad de La Serena, La Serena, Chile 3, 67-84. 